\title{
XII.
}

\section{Behandlungs-Resultate bei complicirten 0torr- hoeen, gewonnen mit Hilfe der kaustischen, resp. galvanokaustischen Methoden}

\author{
von
}

Dr. Jacoby in Breslau.

\section{Serie von fünfzehn Fällen.*)}

Obgleich ich mir nicht vorstelle, dem erfahrenen Collegen im Nachstehenden Neues oder besonders bemerkenswerthes zu sagen, glaube ich doch der Veröffentlichung desselben nicht widerstehen zu dürfen. Es fehlt zunächst, so weit mir die betreffende Literatur bekannt ist, an casuistischen Mittheilungen, geeignet dem angehenden Specialisten als Paradigmen zu dienen. Ferner ist das Vertrauen auf die möglichen praktischen Erfolge, wie sie mit Hilfe der kaustischen Methode unter den fraglichen Verhältnissen sich erzielen lassen, im Vergleich zur therapeutischen Bedeutung des Mittels, soviel ich weiss, noch zu wenig in die betheiligten Kreise gedrungen. Schliesslich will ich nicht verhehlen, dass mit Hilfe des fraglichen Verfahrens im weitern Sinne des Wortes, namentlich bei Complication mit Polypen, doch viel gründlichere und dauernde Resultate erreicht werden, als ich nach den von sehr sachverständiger Seite empfangenen Anschauungen über die definitive Heilbarkeit solcher Zustände durch andere Methoden mir vorzustellen geneigt war. In wie weit diese Thesis sich bewahrheitet, mögen vorurtheilsfreie Kritiker an der Hand

*) Bei den vier galvanokaustiseh behandelten Kranken, deren Geschichte ich im Anfange des $\dot{V}$. Bandes gegeben habe, ist ein Recidiv bis jetzt nicht eingetreten. 
der im Nachfolgenden kurz skizzirten Fälle beurtheilen. Um jede etwaige Unsicherheit auszuschliessen, sind nur solche gewählt, deren Verlauf ich ein bis drei Jahre zu beobachten oder über deren gegenwärtiges Verhalten ich zuverlässige briefliche Auskunft zu erlangen im Stande war. Beobachtungen aus dem letzten Jahre sind absichtlich ausgeschlossen.

\section{I.}

Chranische Otitis media purulenta dextra, complicirt mit oberer Perforation des Trommelfells, polypösen Granulationen $u, s, w$.

Fabrikaufseher Herrmann von bier, $34 \mathrm{Jahr}$ alt, von nieht besonders krältiger Constitution meldet sich am 12 Juni 1869 wegen einer vor drei Wochen äberstandenen linksseitigen Otitis media, von der eine grosse Perforation in der obern Hälfte des Tronmelfells und bis an dieselbe vordringende Schwellung der Labyrinth-Wandschleimhaut mit reichlicher Otorrhoe zurückgeblieben ist. Rechts existirt als Folge eines vor angeblich drei Jahren abgelaufenen analogen Processes gleichfalls an der obern Hälfte eine grosse Perforation, durch welche das Trommelfell fast vollständig bedeckend polypöses Granulations-Gewebe aus dem Jnnern der Trommelhöhle herausgewachsen ist. Während ich den linkseitigen Process mút Hilfe concentrirter Argentum-Lösungen unter allmäligem Verschwinden der genannten Symptome innerhalb der nächsten drei Wochen behandelte, wurde rechts am 17. Juni nach ziemlich fruchtioser Anwendung der Wilde'sehen Sohlinge zưm ersten Male galvanokaustisch mit Hilfe eines ziemlich grossen Cauter's verfahren. Wiederholung desselben Verfahrens am 7. Juli, nachdem inzwischen mehreremal oberfächlich mittels Lápis touchirt war, demnäohst am 23. Juli und noeh viormal innerhalb der folgéden zwei Monate. Trotz sehliesslicher Anwendung verschiedener Adstrigentien war es zur Zeit der Entlassung noch nicht za einem absoluten Yersiegen des pur. Seerets rechterseits gekommen. Zuletzt sah ick den Kranken am 10. Juni 187a. Beiderzeits, existirte im obern Drittheile des Trommelfells eiue ziemlich umfangreiche Perforation; rechts war der untere Abschnitt desselben durch Narbengewebe ersetzt. Von Granulationggeweben war weder äusserlich noch durch die Perforation etras zu entdecken. Purulentes Secret war zur Zeit der Untersuchung nicht vorhanden, wird aber angeblich wenn auch nur in sehr geringer Menge noch periodisch entieert. - In functioneller, Beziehung hatte. Patient soviel gewonnen, dass er mässíg laut gesprochene Worte abgewandt auf 5-6! Entfernung deutlich vernahm, während er vor der Behandlung nur etwa auf einen Fuss Entfernung sehr laut Gesprochenes percipirte. Eine Spaltung des Trommelfells von der Perforation aus zu unternelimen, sah ich mich um so weniger bemutssigt, als sich'bef der 'narbigen Degeneration und Yerdickung des Gewebes ein wahrscheinlleher Gewinn dureh das fraglielle Vorfahron kaum vorausehen liess.

II.

Otitis media (?) chronica purulenta mit polypösen Granulationen in der Tiefe des Gehörganges.

Fräuleịn R, von hier, schwächlieher Constitution, 30 Jahr alt, meldet sich am 1. April 1869 , hat im 8 . Lebensjahre Masern gehabt und seit dem linkseitige, im Allge- 
meinen mässige, während der Menses etwas reichlichere Otorrhoe, nur periodische mässige Schmerzen in der Tiefe des Ohres, nur mässige periodischie subjective Geräusche. Hört 1. Uhr $1^{\prime \prime}$ bei normaler Knochenleitung fïr dieselbe, nur laute Zablen abgewandt anf $10^{\prime}$ und Stimmgabel links. Die Untersuchung. mittels Reflector's ergiebt mässige trichterförmigc Verengerung der Gehörgangslichtung durch. Verdixkung der Haut in dem tieferem Abschnitte desselben und polypöses Granulatious-Gewebe an der hintern, untern und oberen Wand, so dass vom Trommelfell nichts zu selien ist.

Da eine Entfernung des polypösen Gewebes dureh. Wilde, Schlinge unmöglioh war; wurde dasselbe am 6., 10., 17,; 21. April mit entsprechenden Gauteren galvanokaustiseh zerstört, und ein gleiches Verfahren am 1., 4, 15, 22. Mai, am 3. und 13. Juli wiederholt. In der Zwischenzeit wurde an der. Mehrzahl der Tage nur dureh Wasser-Jnjection purificirt, intereurrent, resp. zum Schluss einzelne Adstringentia angewandt und die Kranke am 8, Oktober geheilt entlassen. Sie war frei von' jeder, Spur purulenten Secrets, ron Schmerzen, subjectiven Geräuschen Granulations-Gewebe. Das Trommelfell war yollständig. erhalten, durch Epidermis partiell verdiekt, an seinem untern Absehnitte narbig degenerirt. Bẹ der letzten Untersuchung im Frühlinge d. J. war manubr. m. vollständig șichtbar, sie hörte die Uhr circa $6^{\prime \prime}$ und mässig leise Flihsterzahlen. auf $10^{\prime}$ und etwas: mehr. Es hatte also eine wesentliche Hörverbesserung stattgefunden, die der Kranken auch im geselligen Verkehr deutlich zum Bewusstsein kam.

\section{III.}

\section{Chronische Otorrhoe, complicivt mit einem Polypen in der Tiefe des Gehörganges bei vollständig erhaltenem Trommelfell.}

Lehrer Büttner von Polnisch-Hammer, kräftiger Constitution, 21 Jahre alt, leidet seit etwa 10 Jahren an beständigem linkseitigen eitrigen Ausfluss und Schwerhörigkeit in Eolge von Masern. Hört Uhr l. beim Anlegen an die Ohrmüuschel, uñd laute Zahlen abgewandt anf $10^{\prime}$, Stimmgabel links.

Die hintere Halfto des m. :a ist mit Granulations-Gewebe exfüllt, das eine papilläre Oberfä'be hat, und nur an der innern Wand der Sonde einen freien Eintritt gestattet.

Am 29. Mai 69 wurde mit Hilfe der Schlinge soviel sich fassen liess entfernt, der erhebliche Rest intensiv vielfach galvanokaustisch eauterisirt und dem Kranken tägliche Purification durch Wasser-Injection empfohlen. Unmittelbar nach der Operation hörte der Kranke die ohr bereits $6-7^{\prime \prime}$ und viel weniger late Zahlen als vor derselben. Am 6. Juli ist purulentes Secret nicht mebr vorhanden und der Polyp auf ein Minimum ge schrumpft. Ord. Sol. pl. acetici $0,3-30,0$ täglich zu instilliren und durch Injection zu purificiren. Am 23. Juni ist von Polyp keine Spur mehr sichtbar, der Kranke hört Uhr $2^{\prime}$ und leise Flüsterzahlen auf $10^{\prime}$ sicher. Der Trommelfellbefund ist nicht notịt, doch war dasselbe, wie ich mich deutlich erinnere, vollständig erhalten und auffallend wẹig anomal, so dass es sich hier höchst wahrseheinlich urn einen rom $m$. a. ausgegangenen Polypen handelte. Spätere Nachricht hat die Dauer der Heilung bestätigt.

\section{IV.}

Otitis media chronica purulenta, combinirt mit grosser vorderer Porforation und reichlichem polypösem Granulationsgeibebe.

Pauline Sehröter, 14 Jahre alt, kräftiger Constitution, Stellenbesitzertochter aus Gross-Raake, leidet seit drei Jahren an linkseitiger reichlicher fötider Otorrhoe, Schwer- 
hörigkeit, Temporal-Schmerzen und periodischem Schwindel; ist in Uebrigen gesund. Sie hört Uhr l. einen Zoll gut vom Sehädel aus, nur laute Zahlen auf wenige Fuss Entfernung, Stimmgabel 1. Das linkseitige Trommelfell ist mit grosser vorderer Perforation versehen, die zur Zeit der ersten Untersuchung mit Granulations-Gewebe, das noch einige Linien in den m. a. hineinragt, erfüllt ist. Am 18. Juni 1870 wurde dasselbe zum ersten Male galvanokaustisch zerstïrt, am 13., 17. Juli und 2. October ebenso verfahren. In der Zwischenzeit wurden die zurückgebliebonen Reste ziemlich oft durch Lapis; seltem ner mit concentrirter Chromsäure-lïsung cauterisirt (da mit Rüeksicht auf die in Folge des Krieges mangelnde Assistenz das galvanokaustisehe Verfahren in dieser Zeit nicht regelmässig geübt wurde) und die Patientin am 3. Dezember geheilt entlassen. Innerhalb der letzten Monate vor ihrer Entlassung hatte sie von concentrirter Neilosung Gebrauch gemacht. - Am 2. Juli 1872 meldete sich die Kranke, weil seit Kurzem sich wieder etwas purulentes Secret gezoigt hatte. Die objective Untersuchung ergiebt eine stecknadelkopfgrosse Granulation im Grunde des oberen Abschnittes der grossen vorderen Perforation * Nach zweifacher intensiver Cauterisation mïttels Lapis und demnächstiger Instillation concentrirter Bleilosung ist beides, Granulation und Otorhoe verschwunden. Bei der letzten Untersuchung am 8. Aug. d. J. zeigt sich die vorhandene obere Trommelfellhälfte mit dem Promontorium verwachsen. Die Patientin bört U. 4-5" und ziemlich leise Flüsterzahlen auf 12-15t Entfernung, hat also eine wesentliche Perceptions-Besserung erfahren und ist frei von Schwindel and Schmerzen.

V.

Beiderseitige Otitis media chronica purulenta, rechterseits mit vollständigem Verlust des Trommelfells, der Gehörknöchelchen, Atrofie des Acusticus und seh" geringer Otorrhoe; linkerseils mit sehr reichlicher Otorrhoe und stark entwickellem Granulations-Gevebe.

Fräulein Lina Pächer, Schneiderin von hior, 40 Jahre alt, mittlerer Constitution, leidet seit der Kindheit in Folge von Scharlachfleber an beiderseitiger, namentlich links, reichlicher fötider Otorrhoe und hoclggradiger Schwerhörigkeit. Sie hört Uhr r. nirgends; 1. beim festen Andrücken an die Ohrmuschel und in der Temporal-Gegend. Zallen: $r$. nur ausserordentlich laute in nächster Năho des Ohres so unsicher, dass die Hörfaligkeit hier als geschwunden gelten darf; 1. sehr laute auf $1-2^{\prime}$ zugewandt; Stimmgabel lintis; hat keine Ohrschmerzen aber mehr weniger intensives, von $r$. über den ganzen Kopf sich verbreitendes subjectives Geräusch, fast beständig Benommenleit des Kopfes, oft mehrere Tage Kopfschmerzen und häufig Schwindel. Im l. Gehörgange sieht man eine mit Eiter beschlagene rothe Granulations-Masse, die etwa das letzte Drittheil erfüll. $R$. ist der eitrige Beschlag in der Tiefe des m. a. sehr spärlich und hinter ihm gleichfalls spärliches roth lenchtendes Granulations-Gewebe der Labyrinth-Wand sichtbar.

Am 13. September wurde zum ersten Male die linkseitige Polypen-Masse galvano-

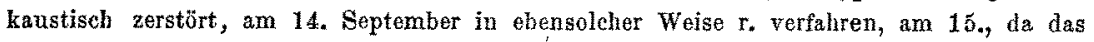
Verfahren am 13. der Anwendung der Wilde'sehen Schlinge den Weg gebalnt hatte, mittels dieser soviel möglich entfernt und am 20. die letzterwähnte Methode wiederbolt. Am 22. September wurde I. intensiv mit Lapis tonchirt, was beiläufg mit nachhaltigen Schmerzen verbunden war. Bis zum 22. Mai 1870 wurdo l. nocl siebenzehn Mal nur galvanokaustisch verfahren und von da $a b$, abgesehen von rereivzelten Anwendungen von Lapis vorzugsweise von verschiedenen adstringirenden Lösungen, namentlich $1-2$ pro- 
centiger von Carbol-Schwefelsaurem Zink Gebrauch gemacht. Auch im Jahre 1871 und 72 hat die Kranke mannigfaltige Adstringentia, insbesondero starke Bleilösungen benützt und ist hierdurch $\mathbf{r}$ : absolut, 1. : bis auf ein Minimum von seröser Secretion frei. L. hört man bei Valsalva'schen Versuch zischendes perforations Geräusch. R. ist die Hörlosigkeit unverändert. L. hat die Kranke namentlich für sprachlichen Verkehr nach ihrer eigenen und der Aussage einer Freundin, die den ganzen Tag um sie ist, wesentlich an Hörfähigkeit gewonnen. Die letzte Untersuchung im Juli d. J. ergab Folgendes: Uhr 1.: etwa $1 / 4^{\prime \prime}$ vom Ohre und mässig laut Zahlen abgewandt auf etwa $15^{\prime}$ Entfernung. Das subjective Geräusch ist seit langer Zeit sehr gering, im Kopf fühlt sie sich ganz frei, Schwindel ist absolut verschwunden. Mittels Reffector's sieht man an Selle des Trommelfells Narbengewebe und an dessen hintern obern Abschnitt eine ganz kleine Perforation, aus der sich nach Angabe der Patientin selbst innerhalb acht Tagen kaum ein Tropfen entleert.

\section{VI.}

\section{Beiderseitige chronische Otorrhoe, an einer Seite complicirt mit polypösem Granulations-Gewebe.}

Ottilio Hoffmann, 7 Jahr alt, Maschinenmeisterstochter zur Zeit in Pielahütte, ziemlich kraftiger Constitution, stellt sich zum ersten Male vor am 26. October 1869 und erzäblte deren Mutter, dass sie in Folge von Scharlach seit ein par Jahre beiderseitige schmerzlose Otornoe, wahrseheinlich auch periodisch subjective Geräusche babe. Nach sorgfältiger Purification sieht man besonders l. ungefähr das hintere Dritttheil des Gehörganges erfüllerdes Granulations-Gewebe.

Nacbdem die Kranke in den ersten Wochen der Kur mebrfach theils mit Lapis, theils mit Wilde'scher Schlinge behandelt worden war, wurde das erstere Verfahren bei seiner Anwendung am $\tau$. October von so nachhaltigen Schmerzen begleitet, dass man bis auf Weiteres davon Abstand nahm. Am 20. October wurde zum ersten Male galvanokaustisch verfahren, dann wieder Cauterisationen mit concentrirter Chromsäure-Lösung abwechselnd mit Lapis angewandt. Da aber das hyperplastische Gewebe mit auffäliger Hartnäckigkeit sich reproducirte, wurde dasselbe am 3. Januar 1870 in Chloroform-Narkose intensiv galvanokaustisch zerstört. Nachdem hierdurch das Meiste beseitigt war, gelang der consequenten Anwendung der oben genamuten Aetzmittel und der sehon fruher erwähnten adteringirenden Lösungen die vollständige und dauernde Beseitigung des Granulations-Gewebes und purulenten Secrets bis Anfang October 1870.* Obgleich eine specielle Notiz über die cingetretene Hörverbesserung in meinem Journal nicht enthalten ist, erinnere ich mich doch genal, dass eine solehe bei der Entlassung constatirt wurde. Nach einer auf meine brieflieho Anfrage erfolgten Antwort des Vaters vom 9. Juli d. J. ist die Hörfähigkeit'im Vergleich zur Zeit des Beginns der Behandlung sehr bofriedigend (geflüsterte Worte auf drei Schritt Entfernung), von Eiterung nur noch ein höchst unbedeutender Rest vorhanden.

*) Die lange Verschleppung der Behandlung wurde in diesem und einzelnen andern der aufgofübrten Fälle vorzugsweise dureh dịe Unregelmässigkeit in Erseheinen der Kranken herbeigefübrt. 


\section{VII.}

\section{Rechtseitige Otorrhoe, complicirt mit hahnenkammförmigen Polypen.}

Max Milde, Zuchthaus-Inspectorssohn aus Schweidnitz, 14Jahre alt, leidet z. Z. seiner ersten Vorstellung am 6 . October $1869^{\circ}$ an rechtseitiger fötider reichlicher Otorrhoo und seit sechs Wochen an linkseitigem Tubenkatarrh, der bei det folgenden Darstellung unbeachtet bleibt. Er hö̀rt $r$. Uhr nïeht beim Anlegen an die Concha, aber von der Temporal-Gegend, nnr sehr laute Zahlen abgewandt anf $1-1 \frac{1}{2} 2^{\prime}$. Das Verhalten der Stimmgabel ist nicht notirt.

Nach Beseitigung ziemlích grosser Quantitäten käsigen Eiters sieht man in der Tiefo des $r$. Gehörganges, das wrommelfell mehr weniger vollständig verdeckend, besonders stark entwickel an 'der hinteren Hälfto' und etwa ein Viertheil des ganzen m. a. erfullend polypöses GranulationswGewebe, das sich bei Berührung mit der Sonde als hahnenkammartiges blättriges Gebilde manifestirt. In Folge dieser Gestalt gelang die Entfernung desselben mittels Wilde'scher Sehlinge nar unvollständig und würde die häufige Wiederholung dieses Verfahrens dem Kranken augenscheinlich viel Schmerzen bereitet haben. Aus diesem Grunde wurde sofort am 7. October soviel wie möglich mittels eines angemessenen Cauter's zerstört. Am 15. Oetober erkannte man bereits in Folge des allmäligen Abganges der Brandschorfe, dass das Trommelfell zum grossen Theil oder ganz erhalten ist. Am 16. October wurde das galvanokanstische Verfahren wiederholt. Am 19. October erkennt man eine kleine vordere untere Perforation und das Trommelfell in seiner Umgẹbung mässig hyperämisch. Am 24. October wurde in unmittelbarer Nähe des Trommelfells nọch eine restirende knopfförmige Granulation galvanokaustisch zerstört, die noch vorhandenen Reste mittels Lapis cauterisirt und am 8. December der Kranke geheilt entlassen, mit der Weísung," noch längero Zeit von concentrirter Sol. pl. acet. als Ohrwasser Gebranch za machen. Am Tage der Entlassung hört er r, Uhr $4-5^{\prime \prime}$ und halblante Zaklen auf etwa' 15 'Entférnung. - Muthmasslich war die gleichzeitige Anwesenheit eines Nasen-Polypen und chronischen Nasenrachenkatarrh's der Grund, weswegen die Pereeptions-Fähigkeit keine grösseren Fortschritte gemacht bätte. Nach Mittheilungen im Jahre 1870 und einer Untersuchung in Jahre 1871 ist ein Recidiv der Otorrhoe oder Polypen nicht eingetreten unid hat die Hörfähigkeit noch erheblich zugenommen.

\section{VIII.}

Inveterirte linkseitige Otitis media chronica purulenta, complicirt mit einem grossen Polypen etc.

Buchhalter Kynast aus Liegnitz, 27 Jahre alt, deponirt gelegentlich seiner Meldung am 28. December 1869, dass er seit zwölf Jahren in Folge von Masern an linkseitiger Ohreneiterung und Polypen leide $e_{+}$Er hört $r$. normal, 1 , die Uhr nicht beim Anlegen an die Mnschel, nur sehr lante Zahlen abgewandt auf $10^{\prime}$ und Stimmgabel $r$. Der linke Gehörgang ist mit einem rosafarbenen, glatten, von allen Seiten mit der Sonde leicht zu umgehenden, den Quer-Durchmesser des $\mathrm{m}$. a. fast vollständig, den Längsdurchmesser b̉ber die Hälfte erfüllenden Polypen versehen. Nach sorgfältiger Reinigung wurde mit Hilfe der galvanokaustischen Sebneideschlingo, die im Hinblick auf die Derbheit des Polypen-Gèwebes vorzüglicher erschien, in wenigen Secunden ein $4-5^{\prime \prime \prime}$ langes und circa. $3-4^{i \prime \prime}$ diokes Stück entfernt. Abgesehen von einer leichten Ohnmachtanwandlung in Folge des psychischen Eindruckes und einer nach der spontanen Aussage des Kranken 
kaum als Sehmerz zu bezeichnenden Empfindung, die nach zehn Minuten vollständig bereits verschwunden war, ging das Verfahren spurlos vorüber. Bald nach der Operation hörte der Kranke weniger laut intonirte Zahlen. Für Uhr war eine Besserung nicht eingetreten. Zur Bekämpfung einer, schon seit langer Zeit bestehenden Benommenheit des Kọfes wurde abgesehen von zweckmässigem Regime methodischer Gcbrauch kleiner Quantitäten Friedrichshaller Bitterwasser empfohlen. Am 9. Januar 1870 wurde soviel wie möglich van dem restirenden Granulation s-Gewebe galyanokaustisch zerstört; functioneli hat sich nichts wesentliches geändert, Kopf aber ist leichter goworden Bei der dritten Vorstellung des Kranken unterbleibt das galvanokaustische Verfahren in Folge einer nach dem zweiten Verfahren eingetretenen beschränkten Gehörgangsentzündung, (Lymphangitis ?) die unter der Anwendung von Bleiwasser in wenigen Tagen verschwindet. Am 23. Jan. findet die dritte Cauterisation mittels Cauter's statt. Der Kranke lobt spontan seine wesentlich gebesserte Stimmung und Arbeitsfähigkeitt. 'Bei der vierten 'Cáterisation hat Patient, eine, Kmpfindupg auf der Zunge. Am 6.,20., 27, Februar, 6., 20.2, 27 März, 3., 10., 17,2, 24. April, 1., 8., 15., 19., 22. Mai und 6. Juni wurden die übrigen orreichbarep intratympanischen und an der hintern Gehörgangs-Wand sitzenden Reste von Granulations-Gewebe galvanokaustiseh, zerstört. Am 19. Juni referir der Kranke, dass in den letzten zehn Tagen das purviente Secret auf ein Minimum reducirt ist. Dio an demselben Tage angestellte Untersuchung bestätigt die Permeabilität der Tưba und fast vollständige Freiheit des Trommelhöhlenraumes von eitriger Flüssigkeit. Trotz der vielfachen (zwanzigmaligen) galvanokaustischen Behandlung hört dor Kranke eine grössero Tasclenuhr ungefär $1 / 2$ " vom Ohre und Flüsterzablen, aber nicht gerade loise gesprochen, auf $10^{\prime}$ Entfornung abgewandt. Am 14. August berichtet Patient: dio Eiterung par stets so gering, dass die, in den Ohren getragene Watte selten befeuchtet war. Austuss hat niemals mehr stattgefunden. Nachdem eine an der Labyrinth-Wand noch vorhandene Verdickung am 14. August und 11. September zuerst mittels Chromsäure-Lösung dás zweite Mal mittels Lapis oberflächlich touchirt war, wurde der Kranke mit der Weìsung, noch längere Zeit von Bleilösung Gebrauch zu machen, entlassen.

Nach einer brieflichen Mittheilung desselben yom 8. Juli 1872 ist eine Versehlimmorung des Zuștandes seit der Entlassung ans der Behandlung nicht eingetreton. Ein mässiges Quantum wässeriger Absonderung ist noch vorhanden.

\section{IX.}

\section{Linhseitige Otorrhoe mit reichlichem Granulations-Gewebe.}

Annà v. Stachelsky, 13 Jahro alt, Sehneidermeisterstochter von hier, leidet am 20. April seit angeblich einem Jahre an linksseitiger Otorrhoe, die schmerzlos entstanden innerhalb der letzten sechs Wochen mit mässigen Obrensclimerzen und periodischem Blutverlust aus dem Ohre sich verbunden haben soll. Abgesehen von linkseitigem Kopfschmerz und periodischen Schwindelanfällen wàr die Pàtientìn gesund. Sie hört 1. Uhr nicht beim Anlegen an die Muschel, nar lante Zahlen auf $10^{\prime}$ und Stimmgabel $L^{\prime}$ Dio intersuchung mittels Reffector's ergiebt in der Tiefe des $m$; $a$ e eine bei. Sondenberührung leicht blutende ziemlich dicke Schicht ron Granulations-Gewebe: Das Trommelfell ist bei der ersten Untersuchung nicht sichtbar. Vom 21. April bis 4. Juni wurdo das hyperpiastsiche Gewebe in sechs galvanokaustischen Sitzungen und durch intercurren te Cauterisation mittels Lapis vollständig zerstört, und der Rest von purulenter Secretion mittels verdännter Chromsäure-Lösung und Bleiwasser beseitigt. Jo mehr von-dem Granulations- 
Gewebe verschwand, desto deutlicher trat das mit einer hintern untern Perforation ver sehene durch Bindegewebsneubilutung verdickte Trommelfell hervor.

Gelegentlich der am 5. Juli 1872 vorgenommenen Inspection referirt dio frihhere Kranke, dass sie bis jetzt frei von Otorrhoe, Kopfschmerz und Schwindel gewesen sei, die Hörprüfung ergiebt noeh fast dasselbe ungünstige Resultat, wie bei der ersten Untersuchung. Die hintere untere Perforation ist auch jetzt noch durch einen kleinen Hügel von Granulations-Gewebe vorlegt und mit Cerumen bedeckt, hinter dem sich elne geringe Menge käsigen Eiters flndet.

\section{$\mathrm{X}$.}

\section{Inveterinte rechtseitige Otorrhoe mit polypösem Granulations-Gewebe.}

Stellenbesitzer Tiegner aus Buschwiz, 40 Jahre alt, kräftiger Constitution, leidet seit angeblich 15 Jahren rechterseits an Otorrhoe, hört $r$. Uhr nur in nächster Nähe des Ohres, nur laute Zablen abgewandt auf $4-5^{\prime}$, Stimmgabel $r$. Das Trommelfell existirt nur in seiner oberen Häfte, der Raum, welchen die untere sonst einnimmt, beziehentlich der entspreehende der Trommelhöble ist zum grössten Theil duroh Granulations-Gewebe erfüllt. Am 24. Mai 1870 wird dasselbe mittels eines entsprechenden Cauter's intensiv canterisirt. Schon am 29. Mai ist die Eiterung fast versehwunden. Der Rest von Granulations-Gewebe wird mit Lapis touchirt. Am 12. Juni ist weder purulentes Secret noch Granulations-Gewebe vorhander. Zur Nachkur wird Sol, pl. acet. empfohlen und stellt sich ete. Tiegner am 24. Juli als vollständig geheilt vor,

21. Juli 1872. Obgleich purificatorisch nichts seit langer Zeit geschehen ist, findet sich auf der untern Hälfte der mit narbigen Bindegewebe bedeckten Labyrinth-Wand (die obere Hälfte des Trommelfells ist mit der Labyrinth-Wand verwachsen) nur eine ganz dünne Schicht von muco-purulentem Secret, nach deren Abstreifung das so eben bezeichnete Bild erscheint. Im $\mathrm{m}$. a. ist absolut keine Absonderung vorhanden. Der frühere Patient hört Uhr ungefähr $1^{\prime \prime}$ und nicht besonders leise Flüsterzahlen auf 10-12' Entfernung abgewandt; von hyperplastischen Granulationen ist kaine Spur.

\section{XI.}

\section{Beiderseitige Otorrhoe mit hyperplastischem Granulations-Gewebe.}

Adelheid Winkler, Köchin, ziemlich kräftiger Constitution, 19 Jahre aIt, aus Klein--Strelitz bei Schweidnitz ist bei ihrer Meldung am 12, März 1870 angeblich seit dreit Jahren nach Typhus sehwerhörig, bat seitdem beiderseits Otorrhoe, beständig subjective Geräusche und seit dem letzten Winter viel Stirnschmerzen. Sie hört $r$. Dhr beim Anlegen an die Ohrmuschel nicht, nur sehr lante Zahlen zugewandt auf $1^{\prime}, 1$. Uhr $2-3^{\prime \prime}$ und etwas, weniger laute Zahlen; Stimmgabel mehr r. als 1. Knochenleitung für Uhr vorbanden. Von Trommelfell oder Resten desselben sieht man bei der ersten Untersuchung nichts in Folge des den betreffenden und zunächst anstossenden Raum des $\mathrm{m}$. a erfullenden hyperplastischen Granulations-Gewebes. Aber noch während der Behandlung werden grosse centrale Perforationen und die resp. Trommelfells-Reste sichtbar.

Durch sechsmalige galvanokaustische Cauterisation des hyperplastischen Gewebes innerhaib etwa sechs Monaten (die Kranke stellte sỉeh böchst unregelmässig ein) wurde sie so vollständig von ihrer Otorrhoe und Schwerhơrigkeit goheilt, dass die letztere nun- 
mehr ihrer Dienstherrschaft nicht mehr auffällt, während sio z. Z. ihrer ersten Meldung bei mir in Folge ihror Schwerhörigkeit aus ihrer Stellung entlassen wurde.

28. Juni 1872 hört sie beiderseits Flüsterzahlen, obgleích nicht gerade leise gesprochen, auf 5-6 Entfernung und Uhr beiderseits einige Zoll weit; von hyperplastischen Granulations-Geweben oder Otorrhoe ist keine Spur; die centralen Perforationen bestehen mit freion Rändern fort.

\section{XII.}

\section{Beiderseitige inveterirte Otorrhoe mit polypösem Granulations-Gewebe.}

Alois Schirmer, Schuhmachergeselle, 22 Jahro alt, nicht kräftiger Constitution, referirt am 13. Juni 1870, dass er gelegentlich dor vor 17 Jahren überstandenen BIattern (die tiefe Narben im Gesicht hinterlassen haben) Schwerhörigkeit und beiderseitige Otorrhoe überkommen habe. Die Untersuchung bestätigt seine Angaben. L. ist dio Otorrhoe viel geriager als $x$. Von Trommelfell ist $z, Z$. der ersten Untersuchung noch nichts sichtbar, erst entsprechend der Zerstörung des hyperplastischen Grannlations - Gewebes in der Tiefe des $\mathrm{m}$. a. entdeckt man den zuletzt zu erwähnenden Befund. Er hört $r$ Uhr etwa $1 / 2^{\prime \prime}$, I. nieht, beiderseits nur laute Zahlen, auf wenige Fuss Entfernung; Knochenleitung für Uhr vom Schädel aus verhält sich normal; Stimmgabelton localisirt sich nicht in den Ohren.

Sechsmalige galvanokaustische Cauterisation innerhalb der nächsten sechs Monato (Patient als Trainsoldat eingezogen, erschien in sehr unregelmässigen Perioden zur Behandlung) genügten neben der intereurrenten und nachträglichen Anwendung concentrirter Bleilösungen ihn von Otorrhoe und hyperplastischem Gewebe zu befreien. Seine Hörfähigkeit hat nur in soweit gewonnen, dass er lauto Zahlen auf etwas grössere Entfernung als vor der Behandlung hört. R. besteht eine grössere vordere Perforation mit freiem Rande und Perforations-Geräusch bei $V a l s a l v a '$ 'schen Versuche. L. eine centrale mit der gegenüberliegenden Wand verlöthet.

\section{XIII.}

\section{Linkseitige Otorrhoe mit polypösem Granulations-Gewebe.}

Joseph Brasch, 13 Jahr alt, Kaufmannssohn aus Lobsenz, leidet gelegentlich seiner ersten Vorstellung am 3. Mai 1871 linkseitig seit angeblich drei Jahren, wahrscheinlich in Folge von Scharlach an reichlicher Otorrboe. Er hört 1 . Uhr etwa $1 / 2^{\prime \prime}$, nur laute Zahlen abgewand auf $10^{\prime}$, stimmgabel l. Nach sorgfältiger Purifeation ergiebt sich eine ziemlich grosse untere Perforation des Trommelfells, die namentlich an ihrem hintern Absehnitt mit perlförmigen Granulations-Gewebe erfïllt ist.

Nachdem am 5, und 10. Mai jedesmal wiederholt intensiv mit einem entsprechenden Cauter das hyperplastische Gewebe zerstört war, trat unter Anwendung caustischer Argentum-Lösungen Rückgang der noch vorhandenen Schwellung und allmälige Vernarbung ein bis zum 21. Mai. Das functionelle Verhalten hat sich so günstig gestaltet, dass er am 10 . Juli 72 Uhr 2-3' und leise Flüsterzahlen auf 10-15' Entfernung hört. Am Trommelfelle sieht man an Stelle der früheren Perforation Narbengewebe und ist dasselbe für Valsal$v a$ 'sohen Versuch gut beweglich. 


\section{XIV. \\ Beiderseitige Otorrhoe mit reichlichem intratympanischem Granulations- Gewebe.}

August Schäzler, 6 Jahr alt, Tagarbeiterssohn von hier, ziemlich kräftiger Copstitution, leidet am 7. Mai 1871 seit angeblich einem Jabre an indolent entstandener beiderseitiger Otorrhoe. Objective Untersuchung ergab ausser reichlichem purulenten Secret grosse centrale Perforationen, die durch hahbikugelförmiges, die Perforations-Ränder noch ziemlich weit uberragendes Granulations-Gewebe vollständig erfullt waren. Achtmalige galvangkasustische Behandling inherhalb eirès Jahres (Patient erșchíen sehr unregelmässig) und nachträglioher anhaltender Gebrauch vorzüglich concentrirter Bleilösungen genügte, die Otorrhoo und das polypöse Geweje verschwinden zu lassen, resp. eine Vervarbung an der Ausgangstäche desselben herbeizufthren.

Am 18. Juli hört er Uhr $r_{*}$ und $1,1 / 2-1^{\prime \prime}$ und beiderseits Flïsterzahlen auf $10^{\prime}$, aber leise gesprochen unsicher. Beiderseits existirt ejn oberer resp. hinterer Trommelfellrest mit schräg nach hinten gerichteten $\mathrm{m}$. a. Die sichtbare Labyrinth-Wandschleimhaut ist vernarbt.

Gelegentlich einer Besichtigung am 25. Juli 1872 zeigt sich auf dem Boden beider Perforationen abermals ein obernächlicher schleimig-eitriger Beschlag, der möglicherweise einem gleichzeitigen Nasenrachen-Katarrh angehört.

\section{XV.}

\section{Otorrhoe, complicirt mit einem grossen, aus der Trommelhöhle stam- menden Polypen und cephalischen Symptomen.}

Paul Einspänner, 13 Jahre alt, Steinsetzerssoln, krättiger Constitution, bekam, laut Angabe vom 27. Mai 1871 vor circa 3 Jahren unter Erseheinungen einer acuten Mittelohrenentzündung eine bald nach ihrem Eintreten reichliche Otorrhoe. Angeblich haben mässigo Schmerzen und irradiirte in der Sehläfengegend fast ununterbrochen angehalten, bei körperlichen Anstrengungen sich gesteigert und häufig den Schlaf unterbrochen. Schwindelanfalle zum Theil bis zum Umfallen und Verlust des Bewusstseins waren hăufg und subjective Geräusche mehr weniger beständig. Schwere des Kopfes, häulge Nackenschmerzen und alle genannten Symptome bestchen auch bis jetzt fort. Seit acht Tagen sind mässige Blutentleerungen aus dem Ohre (jedesmal etwa ein Theelöffol voll) hinzugekommen. Die funetionelle Prüfung ergibt 1 . Uhr $=0$, nur sehr laute Zahlen auf etwa $10^{\prime}$ und Stimmgabel I. Der linke Warzenfortsatz ist mässig geschwollen, die Haut nicht verfärbt, auf Druck mässig empfindlich, geschwollene Lymphdrüsen oder sonstige suspiciose Erscheinungen nicht worhanden. Der Porus acusticus ist $z$. Z. mit angetroeknetem Blute exfull. Nach Ausspritzung desselben sieht man einen den Gehörgang mit Ausschluss des vorderen Drittheils erfullenden blauroth gefärbten nicht verwachsenen Polypen, der sick ziemlich derb anfühlt. Derselbe wurde sofort durch die Wilde'schen Schlinge möglichst weit entfernt und schoo hierdurch für die Zahlen-Perception einige Erleichterung geschaffen. Zwei Tage später referirt der Kranke, dass er seit der Operation weder Schmerzen noch Schwindel, aber guten Schlaf gehabt habe. Am 30. Mai wird eine in der Tiefo des $m$, a, nahe der Insertion des Trommelfells vorhandene schmerzlose Wulst von Granulations-Gewebe galvanokaustisch zerstört; am $\check{\text { b }}$ und 6. Juni solches Gewebe mittels Wilde'scher Schlinge entfernt und am 8. Juni soviel wie 
möglich von dem intratympanischen Polypen-Reste galvanokaustiseh cauterisirt. Nachdem in den nächsten Wochen theils von Lapis, theils von concentrirter Chromsäure-Lösung Gebrauch gemacht worden war, wurde am 11., 19., 23., 26. Juli, 4. und 12. August soviel möglich von dem intratympanischen Gramulations-Gewebe durch entsprechende Cauteren entfernt. Der Entfernung des Restes entzog sich der Kranke bisher, weil angeblich regelmässig bei Berührung des intratympanisehen Granulations-Hägels Schwindelgeftïhl eintrat.

\section{$\mathbf{R} \mathbf{e} \mathbf{u} \mathbf{l}$ t a $\mathrm{t}$ e.}

Ist auch die Zabl der obigen Beobachtungen selbstredend viel zu klein, um daraus massgebende Principien iber den therapeutischen Werth der verschiedenen Methoden abzuleiten, so geht daraus doch unzweideutig hervor:

1. Das galvanokaustische Verfahren hatte in keinem der obigen Fälle eine erhebliche, schmerzhafte oder entziindliche Reaction zur Folge.

2. Dasselbe ist auch unter den desperatesten Umständen (vide Fall V) noch mit günstigem Erfolge bezinglich der Verbesserung der Function in Gebrauch gezogen worden.

3. Es hat da, wo die Kranken sich regelmässig zur Behandlung einstellten, in einer relativ kurzen Zeit das byperplastische Gewebe zerstört und die Otorrhoe definitiv beseitigt.

4. Den sub 1-3 angeführten Resultaten gègenüber kommt die mit der Anwendung desselben verbundene. Umständlichkeit nicht in Betracht.

5. Behufs exacter Würdigung der verschiedenen möglichen Behandlungs-Methoden wären Publicationen über analoge Fälle, die nur mit gowöhnlichen Aetzmitteln behandelt wurden, dringend zu wünschen. 